\title{
Counting backward during chess move choice
}

\author{
DENNIS H. HOLDING \\ University of Louisville, Louisville, Kentucky
}

\begin{abstract}
Backward counting was used to interfere with working memory during tree search from chess game positions. Skilled players at different levels of expertise analyzed games while counting or not, and also while moving the pieces or not. Counting had the effect of reducing the maximum depth of search, the number of branches searched, the total number of moves considered, and the quality of the moves. Moving the pieces increased the depth of search at the expense of alternative branches slightly, but with no reliable effect on move quality. Level of playing skill was correlated with move quality and evaluation accuracy, and interacted with both counting and moving such that stronger players were more, rather than less, affected by counting interference. Such interference seems more likely to affect the central executive than the verbal or visuospatial components of working memory.
\end{abstract}

Although chess has the appearance of a highly visuospatial activity, there are a number of reasons for believing that verbal processes are crucially involved in conducting the game. Players are accustomed to encoding and remembering the moves in English Descriptive (e.g., Q-KB3) or in Algebraic (e.g., Qf3) notations, and their book knowledge concerning the openings, like many middlegame themes and endgame techniques, is organized around verbal labels (e.g., Sicilian defense; minority attack; square of the King). Given this basis of verbal knowledge, it is relevant that a biographical survey of chess titleholders (Elo, 1978) shows evidence that these players possess superior verbal abilities, as represented by a high incidence of professional writing occupations and mastery of foreign languages.

The verbal background of chess principles possessed by all serious players has been clearly implicated as contributing to their standard of practical play. Pfau and Murphy (1988) administered a 75-item multiple-choice test covering all phases of the game, finding that knowledge of this type correlated highly with tournament ratings and proved a better predictor of skill than did a test of memory for visually presented positions. The memory test failed to add significantly to the contribution of verbal knowledge in a multiple-regression equation, despite the role assigned to memory in theories of chess skill.

Because skilled and unskilled chessplayers differ in shortterm memory for briefly exposed game positions, Chase and Simon (1973) put forward the hypothesis that chess skill depends on a repertoire of memorized patterns. Such a repertoire would supply labels for temporary storage in short-term memory, as Jongman (1968) suggested, and it could also provide a set of associations between recognized patterns and plausible moves. The basis of chess skill was therefore seen as depending on memory of specific patterns. There are many objections to this theory, includ-

Correspondence may be addressed to Dennis H. Holding, Department of Psychology, University of Louisville, Louisville, KY 40292. ing the demonstration by Charness (1981) that memory scores may vary when skill is held constant, and by Holding and Reynolds (1982) that skill may vary when memory remains constant. In addition, Holding (1989) has pointed out that the reported differences between players at higher and lower levels of skill are at least as great in the memory of verbally dictated move sequences as in the short-term memory of visually presented positions.

The accumulated evidence reviewed by Holding (1985) suggests instead that chess skill depends on the efficiency of operations on the search tree. Skilled players have been found superior in depth of search, in the use of search algorithms, and in the accuracy of the evaluations that must be returned from end points in the search process. Holding and Pfau (1985) found that higher rated players are consistently superior at anticipating the consequences of projected lines of play, and Holding (1989) has extended the analysis to large binary trees, showing that stronger players improve their predictions as they progress through a tree while weaker players do not.

The task of conducting a tree search during move choice gives ample scope for the employment of memory processes other than those postulated by the patternrecognition theory. Long-term memory, often in the form of verbal knowledge, must come into use during the openings and must play a part in evaluation and in the choice of plausible continuations. At the same time, short-term storage in the form of working memory must serve to hold a number of the intermediate results of the search activity. The player undertaking a search must temporarily store each move in each line that is explored, keeping track of successive changes in the positions, must remember which lines in the tree have been explored, and must remember the outcomes of each line of play.

If working memory is heavily engaged in temporary storage, while at the same time receiving multiple inputs from long-term memory, the inhibition of its operation should produce substantial disruption of the search process. The best-known technique for this purpose is to require subjects to count backward by threes. This task is relatively 
demanding, and, given the analyses of Baddeley and Lieberman (1980), it might be expected to impair the functioning of the central executive component of working memory, regardless of any specific effects on subsidiary mechanisms. There are, however, no experimental precedents for examining interference effects within the search tree.

Players are normally limited to the use of internal representations of the tree of chess variations during tournament games. However, external representations are commonly used during "post-mortem" analyses of games, when players are free to move the pieces. Piece movement is therefore adopted here as an experimental condition, so that the complete experiment includes the four conditions resulting from counting backward or remaining silent, crossed with moving the pieces or remaining still. In this experiment, the potential interaction between the presence of interference and the locus of search representation is thus examined.

The simplest prediction might be to assume that the interference due to counting backward should have its greater effect on internally represented search. However, even in the externalized search, the players need to keep track of what has developed, and they must presumably interface their activities with long-term knowledge, so that counting should continue to cause appreciable disruption of the search. Furthermore, moving the pieces may introduce other complications, leading to disparate effects on different measures of search performance. Although the maximum depth of search might be greater when external representation is employed, the activity of moving the pieces might itself slow down the process of analysis, thus reducing the number of branches explored or changing the overall shape of the game tree.

A final consideration is the level of playing skill. If chess processing becomes more automatic as skill increases, in accordance with the progression described by Fitts (1964) or Shiffrin and Schneider (1977), the interference generated by the counting task should have less effect on the stronger players than on the weaker ones, whose processing should be more controlled and elaborate. A comparable effect was obtained by Milojkovic (1982), who found that experts showed a reduced dependence on spatial separation in visual chess judgments, presumably because their processing was relatively automatic. However, the visual judgment task has requirements very different from those involved in move choice. If practical chess skill is not automatic, but essentially demands more voluminous processing, the reverse effect should be predicted. Stronger players should accordingly be more, rather than less, susceptible to interference during search.

\section{METHOD}

\section{Subjects}

Skilled players with known tournament ratings were individually recruited from local chess clubs. The standard United States Chess Federation (USCF) rating system (Elo, 1978) provides a scale with a mean at 1500 and a standard deviation of 200 points, divided into classes at 200-point intervals from 1000 upwards. All 16 of the players tested were above average on this scale, with ratings varying from 1550 to 2141 , yielding an overall mean of 1830 . Half of those tested were rated below 1830 (mean, 1664) and half above (mean, 1997).

\section{Materials}

A standard tournament board with Staunton chessmen was used to present one practice position and four test positions. The positions, listed below in Forsyth notation, were selected from among those given extensive analysis by Littlewood (1974). The notation records positions rank by rank, from the eighth rank down, separating ranks by commas. Lowercase letters represent Black pieces, uppercase White, and numerals indicate intervening spaces. The practice position was taken from Pedersen-Gallmeyer (Denmark, 1971), after Black's 12th move: Test Position A. Ljubojevic-Donner (Mallorca, 1971); White to move: 5rk, pbq1r1bp, 1p2p1p1, 2ppN3, 3P1PQ1, 2P1B3, PP4PP, 3RR1K1. White won. Test Position B. Hoen-Timman (Stockholm, 1972); White to move: 4rrk1, 6qp, b1np2p1, 2pN4, 1p1nP1P1, 1P5P, 2PQ1RB1, 3N1RK1. Black won. Test Position C. Planinc-Mestrovic (Cacak, 1970); Black to move: 2r1rk1, 1pp1npp, p2bqn2, 4p3, 1PP1P3, P3NBP1, 1BQ2P1P, R4RK1. White won. Test Position D. Tal-Spassky (Tbilisi, 1965); Black to move: r2qrbk1, 1bpn1pp1, 5n1p, 1p1ppN2, 1P2P2N, PB1P3P, 5PP1, R1BQR1K1. Black won.

Note that White and Black each had the move in half the positions, winning one of each pair.

A positional evaluation scale, as used in prior research (Holding, 1985), was employed after each game analysis. The scale assumes a score of 10 for the weaker side, whether White or Black, and permits values from 10 to 20 for the stronger side. Verbal anchors are provided (e.g., 16-“won, with best play"), although the subjects were urged to make consistent use of the numerical values.

\section{Design and Procedure}

The four conditions of the experiment (quiet-still; quiet-move; countstill; count-move) were counterbalanced in blocks of four across the 16 subjects. The order of presentation of Games A, B, C, and D was counterbalanced within each block. Hence, each game position and each combination of conditions occurred at each stage of practice.

Each subject first received general instructions and a copy of the evaluation scale, followed by a dress rehearsal consisting of brief exercise in each experimental condition on the practice position. The players were seated as White throughout the experiment. After an opportunity for questions and the correction of any misunderstandings, the test positions were presented. As each position was exposed to view, the players were told the previous move leading to that position. They then had $3 \mathrm{~min}$, timed by a stopwatch, in which to write down the lines of analysis leading to the best move choice in the position, using any convenient notation. The instructions emphasized that multiple continuations were to be considered, and all players were familiar with the idea of a tree of variations.

When counting backward by threes was required (count-still or countmove), the players began from either 574 or 832 , which were alternated in their order for successive subjects. The number of counts was recorded. The players were urged, with incomplete success, to continue the count at the rates achieved during practice. Individual practice rates varied over a range of 15-20 subtractions per minute, depending on mathematical aptitude. In the conditions where piece movement was necessary (quiet-move or count-move), it was compulsory to execute on the board each move recorded in the analysis.

After analyzing each position, the players carried out the evaluation task using the scale mentioned above. A period of up to $30 \mathrm{sec}$ was allowed for reporting whether, and by how much, White or Black held the advantage.

\section{RESULTS}

\section{Search Statistics}

The chess variations recorded by the players were transcribed into problem-behavior graphs, according to the method originated by Newell and Simon (1965), for the purpose of extracting the search dimensions. Three principal measures preserve the majority of the search information. Maximum depth represents the length, in plies, of the longest sequence in the tree. Number of branches is here preferred to "number of episodes," since reinvestigations from the base moves are not recorded; the number of 
OPERATION

Quiet

Count

\begin{tabular}{|c|c|c|}
\hline $\begin{array}{l}\text { Internal } \\
\text { (Still) }\end{array}$ & 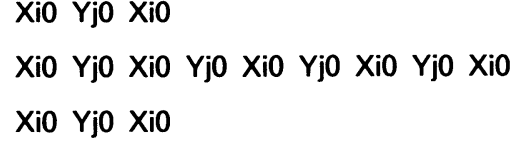 & 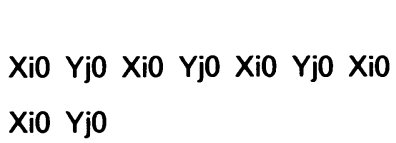 \\
\hline
\end{tabular}

REPRESENTATION

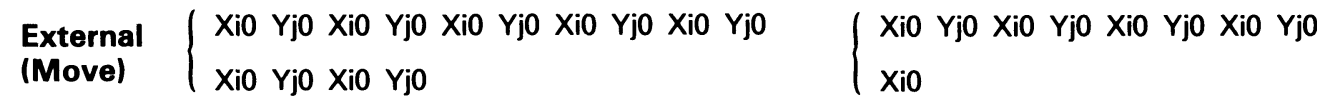

Figure 1. Schematic search trees, showing mean numbers of branches, total moves, and maximum depths, for each experimental condition.

branches refers to the number of divergencies over all plies. Total moves consists of all the moves actually recorded in any tree; there were virtually no implied or null moves.

Analysis of variance showed that, as expected, the maximum depth of search was reduced by counting backward $[F(1,14)=7.79, p<.02]$. The number of branches was substantially reduced $[F(1,14)=22.72, p<.001]$. Similarly, the total number of moves was heavily reduced $[F(1,14)=40.06, p<.001]$. Providing for external representation by moving the pieces produced a different pattern of results. In the movement conditions, the maximum depth was somewhat increased $[F(1,14)=8.48$, $p<.02]$. In contrast, moving the pieces had the effect of reducing the number of branches $[F(1,14)=7.63$, $p<.02]$. The combination of fewer branches with greater depth apparently obviated any significant effect on total moves $[F(1,14)=.04]$. The results for all three statistics in each combination of conditions are represented together in Figure 1, which demonstrates the overall patterns of search, in integer values for the mean scores.

Level of playing skill, which was restricted to aboveaverage players, was not a significant main variable on any search statistic, and no two-way interactions reached significance. However, skill participates in a significant three-way interaction with counting and moving $[F(1,14)=$ $5.15, p<.05]$, arising in the total move scores. The form taken by this interaction, which reflects the overall size
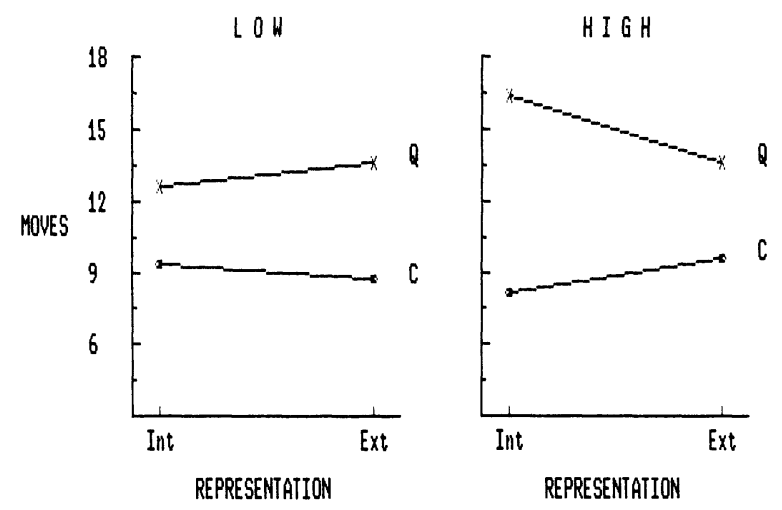

Figure 2. Total moves examined by lower and higher rated players when quiet or counting, with internal or external representation. of the search tree, is shown in Figure 2. Comparison of the curves for players at relatively higher and lower levels of skill suggests that the higher rated players were more affected than the lower rated players by the requirement to count backward. The stronger players appear to have produced more total moves in quiet conditions, but the counting procedure reduced their scores to the level of the relatively weaker players' scores. However, it cannot be shown that the contrast is independently significant $[t(14)=1.28]$. The graph also contains a suggestion that moving the pieces assisted the stronger players when they were counting but the weaker players when they were quiet; yet the effect cannot be considered as reliable separately from the interaction as a whole.

It should be noted that the players seemed incapable of maintaining their count rate while moving the pieces. The obtained rates were somewhat slower than desired in both conditions, but they dropped significantly $[F(1,14)=$ $7.88, p<.02$ ], from 14.4 counts per minute while stillness prevailed to 12.4 while the pieces were moved.

\section{Move Quality}

Although it is important to know how good the chosen moves were, there is no completely objective measure of move quality. The compromise adopted follows earlier research (Holding \& Pfau, 1985; Holding \& Reynolds, 1982 ) in relying principally on authority. Each first move identical with the original grandmaster move (Game A, 1. h4; Game B, 1. Rxf8+; Game C, 1. ... c6; Game D, 1. ... c5) received 2 points; each unflawed move, following the analysis of Littlewood (1974), received 1 point; any poor move, incurring tempo or positional disadvantage but with no material loss, received 0 ; an illegal move was scored as -1 ; a move incurring material loss scored -2 points.

Though somewhat coarse, this scale produced results in broad agreement with the skill levels implied by the USCF rating scale. Thus, summing the quality scores over all four games for each player gave rise to a significant correlation $(r=.67, p<.01)$ between overall score and USCF rating. A related finding was that, again in agreement with prior research (Holding, 1985), the accuracy of evaluation was similarly associated with level of skill. Ideal evaluation ratios for the games (Game A, White 14; 
Game B, Black 12; Game C, White 13; Game D, Black 11) were derived from the Horowitz and Mott-Smith (1973) positional counts. The total evaluation error per player, relative to these estimates, was inversely correlated with playing skill $(r=-.61, p<.02)$.

The effect of skill on move quality was also reflected in the analysis of variance $[F(1,14)=5.31, p<.05]$, with stronger players making better moves. There was an apparent tendency for move quality to improve (from an overall mean of 4.97 to 6.66 ) when the players had to move the pieces, although the effect did not reach significance $[F(1,14)=3.89, p<.10]$. The most important finding concerns the effect of counting backward, which produced a serious deterioration in move quality $[F(1,14)=15.21$, $p<.001]$. The value of the move-quality index was halved when counting was required, dropping from a mean of 7.75 for quiet to a mean of 3.88 for counting.

\section{DISCUSSION}

The results show that the interference produced by counting backward has a strong effect on the search processes involved in move choice. When engaged in counting, all players curtailed their analyses to produce smaller search trees. In addition, their search trees contained fewer branches, and were continued to smaller maximum depths of analysis. Not only did the players explore fewer moves, but the moves that did receive consideration were poorer moves, as is reflected in the quality index. More dramatically, two players with expert ratings gave away their Queens while counting backward. Furthermore, some players were incapable of continuing to count as instructed, either allowing the count rate to fall or catching up in bursts between episodes of chess search. Had it been possible to maintain a fast and steady count rate, it seems probable that the observed effects would have been even greater.

Providing external representation by requiring movement of the pieces seems to distort the search process, making for longer single continuations accompanied by fewer side branches, but with no effect on the total number of moves examined. The fact that moving the pieces decreased the count rate seems to indicate that the task of piece movement imposed its own processing demands, which may have offset any benefit due to externalization. Hence, across all skill levels, moving the pieces did not prove to obviate the interference due to counting, although there appeared to be a somewhat greater effect on the stronger players.

Because the search activity is liable to interference, the activity must take the form of controlled rather than automatic processing. Since the more highly skilled players tend to experience more disruption than the weaker players do, there can be no tendency for increasing automaticity to develop with the acquisition of chess skill. Because any process of move choice based on pattern recognition should be relatively automatic, the experiment therefore supports the conclusion that move choice is based on processes other than pattern recognition. It can be presumed that the focus of the interference with search lies in working memory, which must be responsible for storing lines of search while interfacing with evaluative principles and strategic and tactical knowledge in long-term memory.

The experiment does not provide the information necessary for distinguishing among the components of working memory described by Baddeley (1983), which include a central executive, an articulatory loop, and a visuospatial scratchpad. These components are differentially affected by both the information load and the modality of an interfering task, although the impact of counting backward is relatively nonspecific. Thus, Vallar and Baddeley (1982) confirmed that counting backward by threes produced forgetting of consonant trigrams, presumably by disrupting the central executive; but repeating the word "the" imposed an insufficient load, and a tapping task engaged the wrong modality. Farmer, Berman, and Fletcher (1986) did find specific effects, such that repeating the numbers 1-4 interfered with verbal reasoning, while tapping interfered with a spatial judgment task. Unlike counting backward, both of these tasks imposed relatively light demands, but one interfered specifically with the articulatory loop and the other with the visuospatial scratchpad.
If counting backward affects the central executive, the experimental treatment cannot be regarded as clearly distinguishing between the verbal and spatial aspects of chess processing. Nevertheless, there are prior grounds for expecting the involvement of verbal processes in chess, and it appears likely that the technique of counting backward must have some impact on the articulatory loop subserving verbal rehearsal. Future work should therefore demonstrate some interference with tree search when even a light information load, such as repetitively counting from 1 to 4 , is imposed. It seems unlikely that the simple tapping task used to disrupt the visuospatial scratchpad would have much effect on search processes, since there is at least anecdotal evidence (Hartston, 1976) that some players indulge voluntarily in this very task. Consequently, the extent of any specific contribution by the visuospatial scratchpad to chess processing must remain an open question.

\section{REFERENCES}

Baddeley, A. D. (1983). Working memory. Philosophical Transactions of the Royal Society of London, B302, 311-324.

BADDELEY, A. D., \& Lieberman, K. (1980). Spatial working memory. In R. Nickerson (Ed.), Attention and performance VIII (pp. 521-539). Hillsdale, NJ: Erlbaum.

Charness, N. (1981). Visual short-term memory and aging in chess players. Journal of Gerontology, 36, 615-619.

Chase, W. G., \& Simon, H. A. (1973). The mind's eye in chess. In W. G. Chase (Ed.), Visual information processing (pp. 215-272). New York: Academic Press.

ELo, A. (1978). The rating of chessplayers, past and present. New York: Arco.

Farmer, E. W., Berman, J. V., \& Fletcher, Y. L. (1986). Evidence for a visuo-spatial scratch-pad in working memory. Quarterly Journal of Experimental Psychology: Human Experimental Psychology, 38, 675-688.

FitTs, P. M. (1964). Perceptual-motor skill learning. In A. W. Melton (Ed.), Categories of human learning (pp. 243-285). New York: Academic Press.

Hartston, W. R. (1976). How to cheat at chess. London: Hutchinson HoldiNG, D. H. (1985). The psychology of chess skill. Hillsdale, NJ: Erlbaum.

HoLDING, D. H. (1989). Adversary problem-solving by humans. In K. J. Gilhooly (Ed.), Human and machine problem-solving (pp. 83-122). New York: Plenum.

Holding, D. H. (1989). Evaluation factors in human tree search. American Journal of Psychology, 102, 103-108.

Holding, D. H., \& PfaU, H. D. (1985). Thinking ahead in chess. American Journal of Psychology, 98, 271-282.

Holding, D. H., \& ReYNOLDS, R. I. (1982). Recall or evaluation of chess positions as determinants of chess skill. Memory \& Cognition, 10, 237-242.

Horowitz, I. A., \& MotT-Smith, G. (1973). Point count chess. London: Allen \& Unwin.

Jongman, R. W. (1968). Het oog van de meester. Amsterdam: Van Gorcum.

LITTLEWOOD, J. E. (1974). How to play the middle game in chess. Indianapolis, IN: Bobbs-Merrill.

MiLojkovic, J. D. (1982). Chess imagery in novice and master. Journal of Mental Imagery, 6, 125-144.

Newell, A., \& Simon, H. A. (1965). An example of human chess play in the light of chess-playing programs. In N. Wiener \& J. P. Schade (Eds.), Progress in biocybernetics (pp. 19-75). Amsterdam: Elsevier.

PfaU, H. D., \& MurPhy, M. D. (1988). Role of verbal knowledge in chess. American Journal of Psychology, 101, 73-86.

ShIFFrin, R. M., \& SCHNEIDER, W. (1977). Controlled and automatic human information processing: II. Perceptual learning, automatic attending and a general theory. Psychological Review, 84, 127-190.

VAllar, G., \& BADDEleY, A. D. (1982). Short-term forgetting and the articulatory loop. Quarterly Journal of Experimental Psychology: Human Experimental Psychology, 34, 53-60.

(Manuscript received February 10, 1989.) 